J. Math. Soc. Japan

Vol. 20, Nos. 1-2, 1968

\title{
Algebraic varieties without deformation and the Chow variety
}

\author{
Dedicated to Professor Shôkichi Iyanaga on his 60th birthday
}

\author{
By Goro SHIMURA
}

(Received May 25, 1967)

The purpose of this note is to indicate two simple facts, of which the first one is almost obvious, once it is formulated:

THEOREM 1. If a complex projective non-singular variety $V$ has no deformation, then $V$ is biregularly equivalent to a projective variety defined over an algebraic number field.

Here we say that $V$ has no deformation if $H^{1}(V, \Theta)=0$, where $\Theta$ denotes the sheaf of germs of holomorphic sections of the tangent bundle of $V$. Calabi and Vesentini [1] have proved that $H^{1}(V, \Theta)=0$ if $V$ is the quotient $S / \Gamma$ of an irreducible bounded symmetric domain $S$ of dimension $>1$ by a discontinuous group $\Gamma$ operating freely on $S$ such that $S / \Gamma$ is compact. (See also [4], [5], [11].) Therefore Th. 1 shows that such a quotient has a model defined over an algebraic number field.

To state the second fact, let $C_{p}(N, m, d)$ denote the set of all the Chow points of positive cycles of dimension $m$ and degree $d$ in the projective space of dimension $N$, defined with respect to a universal domain of characteristic $p \geqq 0$. It is well known that $C_{p}(N, m, d)$ is a Zariski closed set in a certain projective space, which is defined by equations with coefficients in the prime field. Then one can ask the following question:

(Q) Is every absolutely irreducible component of $C_{p}(N, m, d)$ defined over the prime field ? ${ }^{1)}$

The answer is negative if the characteristic is 0 :

THEOREM 2. There exist positive integers $N, m, d$ such that $C_{0}(N, m, d)$ has a component which is not defined over the rational number field.

Such a component will be obtained so as to contain the Chow point of a certain variety without deformation. This is why we present these two theorems together. We shall also show in the last section that the answer to the question $(Q)$ is still negative even if the characteristic is positive.

1) I thank S. Lichtenbaum for reminding me of this question. 
1. We shall denote by $c(X)$ the Chow point of a positive cycle $X$ in a projective space, and by $P^{N}$ the projective space of dimension $N$. A variety or a curve will always mean an absolutely irreducible one. In this section, the universal domain is the complex number field $\boldsymbol{C}$.

PROPOSITION 1. Let $V$ be a non-singular variety of dimension $m$ and degree $d$ in $P^{N}$, and $B$ a component of $C_{0}(N, m, d)$ containing $c(V)$. Suppose that $V$ has no deformation. Then there exists a Zariski open subset $B^{\prime}$ of $B$ such that if $c(X) \in B^{\prime}, X$ is a non-singular variety biregularly isomorphic to $V$.

PROOF. Let $k_{0}$ be a finitely generated extension of the rational number field $\boldsymbol{Q}$ over which $c(V)$ and $B$ are rational. Let $x$ be a generic point of $B$ over $k_{0}$, and $W$ the cycle such that $c(W)=x$. We shall now prove

(1.1) $W$ is a non-singular variety biregularly isomorphic to $V$.

The non-singularity is obvious, since $V$ is a specialization of $W$ over $k_{0}$. Put $u=c(V)$. We can find a generic specialization $y$ of $x$ over $k_{0}$ and a finitely generated extension $k$ of $k_{0}$, such that $u$ is a specialization of $y$ over $k$ and $k(y)$ is a regular extension of $k$ of dimension one. Let $E$ be a complete nonsingular curve with a generic point $z$ over $k$ such that $k(z)=k(y)$, and $f$ a morphism of $E$ into $B$, rational over $k$, such that $f(z)=y$. Take a point $v$ on $E$ such that $f(v)=u$. For each $t \in E$, let $W_{t}$ denote the cycle such that $c\left(W_{t}\right)$ $=f(t)$. Then $W_{t}$ is the unique specialization of $W_{z}$ compatible with the specialization $z \rightarrow t$ over $k$. Let $E^{\prime}$ be the set of all $t$ on $E$ such that $W_{t}$ is a nonsingular variety. Then $E^{\prime}$ is a Zariski open subset of $E$ rational over $k$, containing both $z$ and $v$. Let $w$ be a generic point of $W_{z}$ over $k(z)$. (Note that $W_{z}$ is defined over $k(z)$, since $c\left(W_{z}\right)=f(z)$.) Let $H$ be the locus of $z \times w$ on $E^{\prime} \times P^{N}$ over $k$. Then we see easily that the intersection product of $H$ with $t \times P^{N}$ on $E^{\prime} \times P^{N}$ is $t \times W_{t}$ for every $t \in E^{\prime}$. By [10, Ch. VI, $\left.\S 2, T h .6\right]$, every point of $t \times W_{t}$ is simple on $H$. Thus we have a non-singular variety $H$ which may be regarded as a fibre bundle with $E^{\prime}$ as base and the $W_{t}$ as fibres. By the theorem of Frölicher and Nijenhuis [2] (cf. also [3]), our assumption $H^{1}(V, \Theta)=0$ implies that there exists a neighborhood $M$ of $v$ on $E^{\prime}$ such that $V\left(=W_{v}\right)$ is biregularly isomorphic to $W_{t}$ for every $t \in M$. We can find a generic point $s$ of $E$ over $k$ lying in $M$. Let $q$ be a biregular morphism of $W_{s}$ to $V$. Since $f(s)$ is a generic specialization of $x$ over $k_{0}$, there exists an automorphism $\sigma$ of $\boldsymbol{C}$ over $k_{0}$ such that $f(s)^{\sigma}=x$. Then $q^{\sigma}$ is a biregular morphism of $W\left(=\left(W_{s}\right)^{\sigma}\right)$ to $V$. This proves (1.1).

Now for every $c(X) \in B,(c(X), c(V))$ is a specialization of $(c(W), c(V))$ over $k_{0}$. Over this specialization, the graph of $q^{\sigma}$ can be specialized to the graph of a biregular morphism of $X$ onto $V$, at least for all $c(X)$ in some Zariski open subset of $B$ (see for example [9, §12.3, Prop. 24]). This proves our proposition. 
Proof of Theorem 1. Let $V, B$ and $B^{\prime}$ be as in Prop. 1 , and $k$ the smallest field of definition for $B$. Then $k$ is an algebraic number field of finite degree. We can find a point $v$ of $B^{\prime}$ algebraic over $k$. Let $X$ be such that $c(X)=v$. Then $X$ is biregularly isomorphic to $V$ and defined over $k(v)$. This proves Th. 1.

One could actually prove Th. 1 more directly. For example, take the locus $U$ of $c(V)$ over the algebraic closure of $\boldsymbol{Q}$, and construct a fibre variety of which the base is a Zariski open subset of $U$ and the generic fibre at $c(V)$ is $V$. Specializing $c(V)$ to an algebraic point on $U$ close to $c(V)$, one obtains the desired conclusion.

2. The universal domain being still $\boldsymbol{C}$, let $V$ be a projective variety. A subfield $h$ of $\boldsymbol{C}$ is called the bottom field (resp. the strong bottom field) for $V$ if the following condition is satisfied.

(2.1) An automorphism $\sigma$ of $\boldsymbol{C}$ is the identity mapping on $h$ if and only if $V^{\sigma}$ is birationally (resp. biregularly) equivalent to $V$.

Such a field $h$ is unique for $V$ if it exists. The following three assertions are immediate consequences of this definition.

(2.2) If $W$ is birationally (resp. biregularly) equivalent to $V$, and $h$ is the bottom field (resp. the strong bottom field) for $V$, then $h$ is the bottom field (resp. the strong bottom field) for $W$.

(2.3) Every field of definition for $V$ contains the bottom field (resp. the strong bottom field) for $V$, if the latter exists.

(2.4) If $V$ is defined over an algebraic number field, then the bottom field and the strong bottom field for $V$ exist and are algebraic number fields of finite degree.

We say that $V$ is a minimal model if every rational mapping of a variety $X$ into $V$ is defined at all the simple points of $X$. Then we can easily verify

(2.5) The bottom field and the strong bottom field for $V$ exist and coincide, if either one of them exists and $V$ is a non-singular minimal model.

Proposition 2. Let $V$ and $B$ be as in Prop. 1. Suppose that $V$ has no deformation. Then the bottom field and the strong bottom field for $V$ exist and are contained in the smallest field of definition for $B$.

Proof. By (2.2), (2.4) and Th. 1, we know that the strong bottom field for $V$ exists and is an algebraic number field of finite degree. Denote it by $h$. Let $k_{0}$ be a finitely generated extension of $h$ over which $B$ and $c(V)$ are rational. Let $x$ be a generic point of $B$ over $k_{0}$, and $W$ the variety such that $c(W)=x$. By (1.1) and (2.2), $h$ is the strong bottom field for $W$. Let $k$ be the smallest field of definition for $B$. Since $W$ is defined over $k(x)$, we have $h \subset k(x)$ by (2.3). Since both $h$ and $k$ are algebraic over $\boldsymbol{Q}$ and $k$ is algebraic- 
ally closed in $k(x)$, we have $h \subset k$, which completes the proof for the strong bottom field. The same reasoning applies also to the bottom field.

3. In order to prove Th. 2 , it is sufficient, in view of Prop. 2, to show the existence of a non-singular projective variety without deformation whose strong bottom field is not $\boldsymbol{Q}$. We shall obtain such a variety as a quotient $\mathscr{S}^{r} / \Gamma$ of the product of $r$ copies of the upper half plane $\mathscr{S}=\{z \in \boldsymbol{C} \mid \operatorname{Im}(z)>0\}$ by a discontinuous group $\Gamma$. To define $\Gamma$, we consider a totally real algebraic number field $F$ of degree $g$, and a division quaternion algebra $D$ over $F$. Let $p_{\infty_{1}}, \cdots, p_{\infty \mathrm{g}}$ be the archimedean primes of $F$. Suppose that $D$ is unramified at $p_{\infty_{1}}, \cdots, p_{\infty r}$ and ramified at $p_{\infty r+1}, \cdots, p_{\infty \mathrm{g}}$. Put $D_{\boldsymbol{R}}=D \underset{\boldsymbol{Q}}{\otimes} \boldsymbol{R}$. Then $D_{\boldsymbol{R}}$ can be identified with $M_{2}(\boldsymbol{R})^{r} \times \boldsymbol{K}^{g-r}$, the product of $r$ copies of the total matrix algebra of degree 2 over the real number field $\boldsymbol{R}$ and $g-r$ copies of the division ring $\boldsymbol{K}$ of real quaternions. Let $\mathfrak{p}$ be a maximal order in $D$, and $\Gamma(\mathfrak{p})$ the group of all units in $\mathfrak{D}$ whose reduced norm to $F$ is 1 . For every $\alpha \in \Gamma(\mathfrak{D})$, let $\left[\begin{array}{ll}a_{\nu} & b_{\nu} \\ c_{\nu} & d_{\nu}\end{array}\right](\nu=1, \cdots, r)$ be the projections of $\alpha$ to the first $r$ factors $M_{2}(\boldsymbol{R})$ of $D_{R}$. Define the action of $\alpha$ on $\mathscr{S}^{r}$ by

$$
\alpha\left(z_{1}, \cdots, z_{r}\right)=\left(w_{1}, \cdots, w_{r}\right), w_{\nu}=\left(a_{\nu} z_{\nu}+b_{\nu}\right)\left(c_{\nu} z_{\nu}+d_{\nu}\right)^{-1} \quad\left(z_{\nu} \in \mathfrak{S}\right) .
$$

Then $\Gamma(\mathfrak{D})$ gives a discontinuous group operating on $\mathfrak{S}^{r}$. Now we assume

(3.1) The multiplicative group of invertible elements of $D$ has no element of finite order other than \pm 1 .

Then $\mathfrak{S}^{r} / \Gamma(\mathfrak{D})$ is biregularly isomorphic to a non-singular projective variety $V$. By [5, Th. 7.4], $V$ has no deformation if $r>1$. Assume further

(3.2) $\quad r=g-1>1$, and $p_{\infty \mathrm{g}}$ corresponds to the identity mapping of $F$.

The results of $[7,4.9$ and 4.10$]$ tell us that, under the assumptions (3.1) and (3.2), the composite of $F$ and the bottom field for $V$ is an unramified class field over $F$ corresponding to an ideal group $I(D / F)$ in $F$ generated by the following three types of ideals :

(I) the principal ideals (a) such that $a$ is totally positive;

(II) the squares of all ideals in $F$;

(III) the prime ideals in $F$ which are ramified in $D$.

In view of these facts, we construct our counterexample in the following way. Take any totally real algebraic number field $F$ of degree $g>2$ whose class number is even. (For example, $F=\boldsymbol{Q}(\sqrt{10}, \sqrt{13})$.) We can find a prime ideal $\mathfrak{p}$ in $F$ which is generated by a totally positive element and decomposes in every quadratic extension of $F$ containing non-trivial roots of unity. There exists a quaternion algebra $D$ over $F$ which is ramified exactly at $\mathfrak{p}$ and $p_{\infty}$, where $p_{\infty \mathrm{g}}$ is as in (3.2). By our choice of $\mathfrak{p}$, we see that (3.1) is satisfied, and $I(D / F)$ is different from the whole ideal group of $F$. Hence a model for $\mathfrak{S}^{r} / \Gamma(\mathfrak{D})$, 
with any maximal order $\mathfrak{D}$ in $D$, has the bottom field different from $\boldsymbol{Q}$. This completes the proof of Th. 2.

It is possible to obtain many more examples of varieties without deformation and with bottom fields $\neq \boldsymbol{Q}$, by considering the quotient of a bounded symmetric domain, other than $\mathfrak{S}^{r}$, by a discontinuous group. This can be done, for example, by making the results of $[7, \S 2]$ more precise, or extending them to the case of congruence subgroup of the discontinuous group in question (cf. also [8]).

4. 2) Another type of counterexample can be obtained by considering the topological structure of varieties. We first prove an elementary

Proposition 3. Let $V$ and $V^{\prime}$ be non-singular projective varieties whose Chow points belong to the same component of $C_{0}(N, m, d)$. Then there exists $a$ diffeomorphism of $V$ onto $V^{\prime}$.

Proof. Let $B, k_{0}, z, E^{\prime}, f, w$ and $H$ be as in the proof of Prop. 1. We see that $V$, as a fibre of $H$, is diffeomorphic to a generic fibre $W_{z}$ with a generic point $z$ of $E^{\prime}$ over $k$. (Note that we do not need the condition $H^{1}(V, \Theta)$ $=0$.) Let $B_{0}$ be the set of all simple points $r$ of $B$ such that $r=c(X)$ with a non-singular variety $X$. Then $B_{0}$ is a Zariski open subset of $B$ rational over $k_{0}$. Let $K$ be the locus of $f(z) \times w$ over $k_{0}$ on $B_{0} \times P^{N}$. By the same type of argument as in the proof of Prop. 1, we see that $K$ is non-singular, and $K \rightarrow B_{0}$ defines a fibre bundle whose fibres are the varieties $X$ such that $c(X) \in B_{0}$. If $c(X)$ and $c(Y)$ belong to $B_{0}$, then $X$ and $Y$ are diffeomorphic. Since $c\left(W_{z}\right)$ $\left(=f(z)\right.$ ) belongs to $B_{0}$, we see that $V$ is diffeomorphic to the fibres of $K$. Applying the same reasoning to $V^{\prime}$, we obtain our assertion.

Now Serre [6] gave an example of a non-singular projective variety $V$ defined over an algebraic number field $k$ such that $V$ is not homeomorphic to $V^{\sigma}$ for some isomorphism $\sigma$ of $k$ into $\boldsymbol{C}$. Then the component of $C_{0}(N, m, d)$ containing $c(V)$ can not be defined over $\boldsymbol{Q}$, since it can not contain $c\left(V^{\sigma}\right)=c(V)^{\sigma}$ in view of Prop. 3. It may be interesting to investigate the deformation of this $V$.

5. Let us now consider $C_{p}(N, m, d)$ for positive $p$. Since $C_{0}(N, m, d)$ is defined by equations with rational coefficients, we can naturally speak of its reduction modulo $p$ for a prime number $p$.

Proposition 4. There exists a finite set $S(N, m, d)$ of rational primes such that $C_{p}(N, m, d)$ is exactly the reduction of $C_{0}(N, m, d)$ modulo $p$ if $p$ is not contained in $S(N, m, d)$.

This can easily be verified by applying the following two simple principles

2) The possibility of using Serre's example was kindly suggested by G. Washnitzer. 
to the defining equations of $C_{0}(N, m, d)$.

(5.1) Reduction modulo $p$ of the intersection of algebraic sets is the intersection of the reduced sets for all except a finite number of $p$.

(5.2) Reduction modulo $p$ of the projection of an algebraic set is the projection of the reduced set for all except a finite number of $p$.

For more precise statements of these facts, see, for example, [9, Ch. III, Prop. 19, Prop. 20].

Now let $B$ be a component of $C_{0}(N, m, d)$ which is not defined over $\boldsymbol{Q}$. Let $B_{1}, \cdots, B_{r}$ be all the components of $C_{0}(N, m, d)$ with $B_{1}=B$. Let $y_{i}=c\left(B_{i}\right)$ for $i=1, \cdots, r$. Then both $\boldsymbol{Q}\left(y_{1}\right)$ and $\boldsymbol{Q}\left(y_{1}, \cdots, y_{r}\right)$ are algebraic number fields of degree $>1$. We can find a prime ideal $p$ in $\boldsymbol{Q}\left(y_{1}, \cdots, y_{r}\right)$ such that: (i) $y_{1}$ modulo $\mathfrak{p}$ is not rational over the prime field; (ii) $\mathfrak{p}\left(B_{1}\right)^{3)}$ is absolutely irreducible and not contained in $\mathfrak{p}\left(B_{i}\right)$ for $i>1$; and (iii) the rational prime $p$ divisible by $\mathfrak{p}$ does not belong to $S(N, m, d)$. Then $\mathfrak{p}\left(B_{1}\right)$ is a component of $C_{p}(N, m, d)$ which is not defined over the prime field. Thus the answer to the question $(Q)$ is negative at least for infinitely many prime characteristics.

\section{Princeton University}

\section{References}

[1] E. Calabi and E. Vesentini, On compact, locally symmetric Kähler manifolds, Ann. of Math., 71 (1960), 472-507.

[2] A. Frölicher and A. Nijenhuis, A theorem on stability of complex structures, Proc. Nat. Acad. Sci. U. S. A., 43 (1957), 239-241.

[3] K. Kodaira and D.C. Spencer, On deformations of complex analytic structures, Ann. of Math., 67 (1958), 328-466.

[4] Y. Matsushima and S. Murakami, On certain cohomology groups attached to hermitian symmetric spaces, Osaka J. Math., 2 (1965), 1-35.

[5] Y. Matsushima and G. Shimura, On the cohomology groups attached to certain vector valued differential forms on the product of the upper half planes, Ann. of Math., 78 (1963), 417-449.

[6] J.-P. Serre, Exemples de variétés projectives conjuguées non homéomorphes, C. R. Acad. Sci. Paris, 258 (1964), 4194-4196.

[7] G. Shimura, Class-fields and automorphic functions, Ann. of Math., 80 (1964), 444-463.

[8] G. Shimura, On the field of definition for a field of automorphic functions, I, II, III, Ann. of Math., 80 (1964), 160-189; 81 (1965), 124-165; 83 (1966), 377-385.

[9] G. Shimura and Y. Taniyama, Complex multiplication of abelian varieties and its applications to number theory, Publ. Math. Soc. Japan, No. 6, 1961.

[10] A. Weil, Foundations of algebraic geometry, 2nd edition, Providence, 1962.

[11] A. Weil, On discrete subgroups of Lie groups, I, II, Ann. of Math., 72 (1960), 369-384; 75 (1962), 578-602.

3) $\mathfrak{F}(X)$ means the cycle obtained from $X$ by reduction modulo $\mathfrak{p}$. 